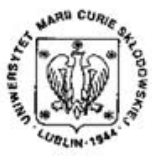

Annales UMCS Informatica AI X, 2 (2010) 133-141 DOI: $10.2478 / \mathrm{v} 10065-010-0059-\mathrm{z}$

Annales UMCS

Informatica

Lublin-Polonia Sectio AI

http://www.annales.umcs.lublin.pl/

\title{
ST-Elevation Myocardial Infarction simulations
}

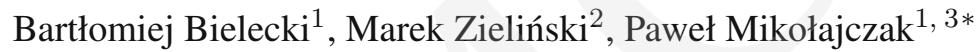 \\ ${ }^{1}$ State School of Higher Education in Chetm, \\ ul. Pocztowa 54, 22-100 Chetm, Poland. \\ ${ }^{2}$ Province Hospital of Ludwik Rydygier in Chetm, \\ ul. Ceramiczna 1, 22-100 Chetm, Poland. \\ ${ }^{3}$ Marie Curie-Sklodowska University in Lublin, \\ Plac M.Curie-Skłodowskiej 1, Lublin, Poland.
}

\begin{abstract}
The authors present the methods of ECG signal simulation especially ST-Elevation Myocardial Infarction known as STEMI. It occurs in the case of blood flow around and towards heart problem. It is strongly associated with a heart attack due to low level of oxygen transported to the heart muscle. The most common symptoms of this disease are chest pain, breath shortness or sweating. There are many symptoms of STEMI but the most characteristic feature is a line change between the QRS complex and T wave which are considered by the authors. The obtained results are compared with actual signals from three leads of ECG Holter, or scanned signals digitalized to formatted files. All simulations, results and analyses are made used of in the applications created by the authors.
\end{abstract}

\section{ECG signal simulation}

Good understanding of wave features allows to create a method for ECG signal simulation. Based on the normal heart conditions examination performed by the basic ECG register, the authors decided to develop a model which contains three different cycles with a possibility of their expansion into long signals. The extended length is needed for a time frequency analysis, but in this model it only multiplies the features of three basic cycles. The simulator changes a signal using mathematical algorithms, generating curves almost in unrestricted amount of

*pawel.mikolajczak@umcs.lublin.pl 
Pobrane z czasopisma Annales AI- Informatica http://ai.annales.umcs.pl

Data: 26/04/2023 16:44:03

outcomes, granting all properties of ECG. This advantage allows to design waves which are characteristic of every lead.

The first stage of the simulation is a baseline generation. If the best method can be found actual signals will be analyzed and it is proved that sinus lines with different wave lengths and amplitudes give the good results. The additional information, related with a baseline and declared by the application user, considers the settings of a distance between $\mathrm{T}$ wave of the previous cycle and $\mathrm{P}$ wave of another one.

The simulation of each wave is based on the straightforward algorithms with the division into two types: linear or Gaussian.

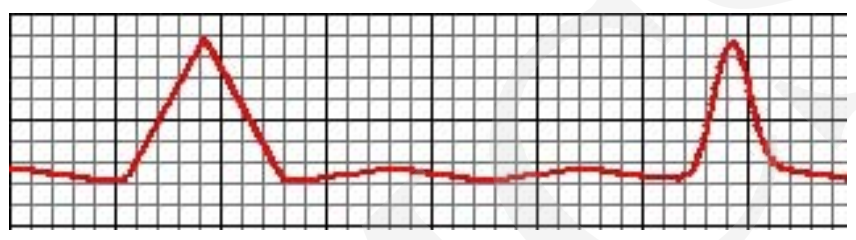

Fig. 1. Two types of waves. The left is linear, and the right is Gaussian.

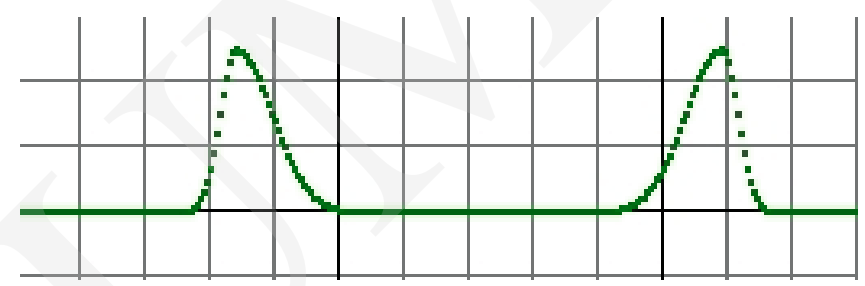

Fig. 2. Two Gaussian waves with different $\sigma$ values.

The linear type mostly represents the waves with small amplitude and a short duration time. They are based on the Bresenham algorithm which is used in the computer graphics.

The Gaussian type is based on the Gausian Curves with some modifications of parameters.

$$
y_{1}(x)=y_{0}(x)+A \exp \left(-\frac{c^{2}\left(x-x_{0}\right)^{2}}{\sigma^{*} l^{2}}\right),
$$

where $y_{1}(x)$ - obtained value, $y_{0}(0)$ - value on baseline, $A$ - amplitude taken from the form, $c$ - value with an influence on a curve shape, $x$ - point belonging to a wave, $x_{0}$ - extremum, $l$ - duration time of a wave, $\sigma$ - parameters with an influence on arm shape.

Every wave is divided into two sections: from the start to peak and from a peak to the end. This solution gives waves with different arms of curves (Fig. 3).

For the generated completed ECG signal, the model dictates loading data in the following order:

- baseline parameters,

- signal length,

- PQ segments, 
- ST segments,

- all three P waves,

- all three P waves,

- all three R waves,

- all three $\mathrm{S}$ waves,

- all three $\mathrm{T}$ waves,

- noise,

- changes of ST segment characteristic of STEMI.

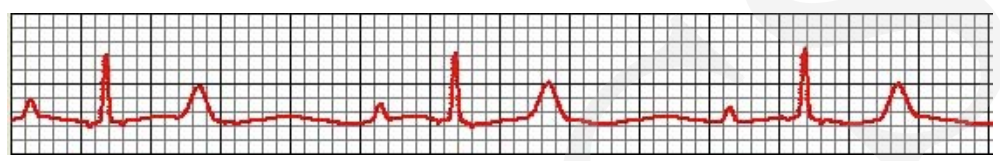

Fig. 3. Pure signal without noise and ST segment changes.

Noise is also an important part of each signal. In common examinations noise is caused by a patient muscles shape, lower temperature in the examination room and equipment failures. Noise appears in most cases in the range of baseline excluding high amplitude waves and there is a very significant drawback in the waves or QRS complex detection algorithms [3]. Both situations are considered in the model as it is shown in Fig. 5 and 6.

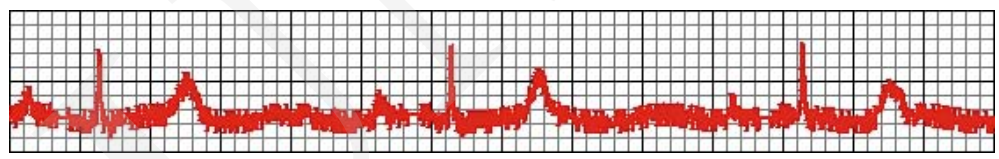

Fig. 4. Higher noise level.

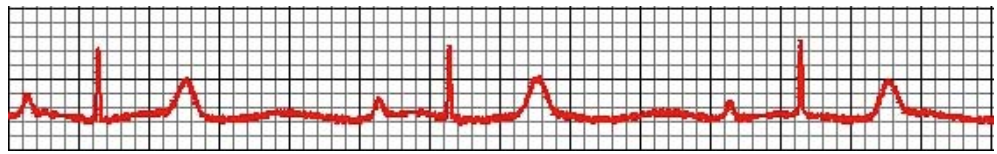

Fig. 5. Normal noise level.

Noise is represented by the formula:

$$
y_{1}(x)=y_{0}(x)+A^{*} Z^{*} C,
$$

where $y_{1}(x)$ - obtained value, $y_{0}(x)$ - input value, $A$ - noise amplitude taken from the application form, $Z$ - random boolean value. If the value is equal to true noise it is directed above the signal but when the value is equal to false noise it is directed below the signal, $C$ random value dependent on the $\mathrm{R}$ wave amplitude.

The most important aspect of, the STEMI simulation is obtaining pathological ST segments which are different from a normal sinus rhythm[4]. ST segments modelling is completely 
diffrent from the above described method because this is generated independent of current waves and only baseline parameters are important here. The start point and the end point of the ST segment have to be taken from the form. Due to the ST segment properties, it belongs to the right part of the R (not S) wave and the left part of the T wave. For the ST wave modelling very good results and easy computation are given by the simple formula:

$$
y=a x^{*} b \sin (x),
$$

where $a$ - value which changes from $0.08 \mathrm{mV}$ to the half amplitude of the next $\mathrm{T}$ wave, $b$-value which changes from $0.08 \mathrm{mV}$ to the amplitude of the next $\mathrm{T}$ wave.

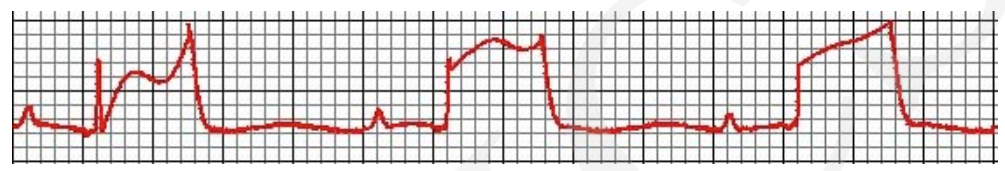

Fig. 6. An example with the ST segment modification. For the comparison the base signal is presented in Fig. 4.

The last stage of the simulation process is joining all single signals, which represent every lead, to one project. It is very important that all clinical features in the project have to satisfy the following conditions. In most cases the time of waves appearance in every lead must be the same. Waves may have different amplitudes and shapes, but it is impossible to carry out all ECG examination without cardiological knowledge. All leads automated joining to one examination is predicted to take place in the future.

\section{Examples}

The application created by the authors was used for the simulation process. It has an interface which allows to create different kinds of signals.

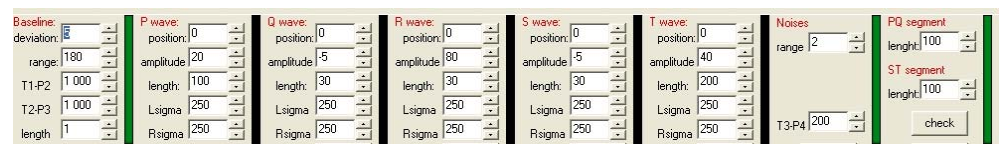

Fig. 7. Main interface of the simulator.

As the ECG manufacturers have their own solutions of data saving, the obtained values can be scaled. The leading Polish producer has output values between $(-50,50)$ and the American one between $(-500,500)$ in files. These values are increased by an amplifier and they do not represent voltage in the output. In the simulator the leads are denoted as: L1, L2, L3 and every signal includes three cycles (A, B, C) and five waves (P, Q, R, S, T). Each signal has its own global values like: dev - baseline amplitude, rng - baseline wave length, TxPy - time between the previous $\mathrm{T}$ wave and next P wave, nss - noise, PQ - PQ segment, Stex-boolean 
value returning true when STEMI exists, STpos - time of ST segment changes, appearance, STamp - amplitude of ST segment. The following wave parameters are given: pos - extremum position (if pos $=0$ then a wave is regular), amp - amplitude, lgt - duration time, Lsg - value $\sigma$, (from expr. 1) of the left side of the curve, $\mathrm{RSg}$ - value $\sigma$, (from expr. 1) of the right side of the curve. Hence: L2APamp $=1$ means that this is the amplitude $1 \mathrm{mV}$ for $\mathrm{P}$ wave from the first cycle, the second lead L2dev $=0.3$ means that this is the amplitude of baseline for the second lead.

\begin{tabular}{|l|l|l|l|l|l|l|l|}
\hline & pos & lamp & lgt & LSg & RSg \\
\hline L1A & $0,0,0,0,0$ & $20,-5,80,-5,40$ & $100,30,30,30,200$ & $250,250,250,250,250250,250,250,250,250$ \\
\hline L1B & $0,0,0,0,0$ & $20,-5,80,-5,40$ & $100,30,30,30,200$ & $250,250,250,250,250$ & $250,250,250,250,250$ \\
\hline L1C & $0,0,0,0,0$ & $20,-5,80,-5,40$ & $100,30,30,30,200$ & $250,250,250,250,250$ & $250,250,250,250,250$ \\
\hline L2A & $0,0,0,0,0$ & $66,-5,66,-5,40$ & $100,30,30,30,200$ & $250,250,250,250,250250,250,250,250,250$ \\
\hline L2B & $0,0,0,0,0$ & $66,-5,66,-5,40$ & $100,30,30,30,200$ & $250,250,250,250,250$ & $250,250,250,250,250$ \\
\hline L2C & $0,0,0,0,0$ & $66,-5,66,-5,40$ & $100,30,30,30,200$ & $250,250,250,250,250250,250,250,250,250$ \\
\hline L3A & $0,0,0,0,0$ & $20,-5,80,-5,40$ & $100,30,30,30,200$ & $250,250,250,250,250250,250,250,250,250$ \\
\hline L3B & $0,0,0,0,0$ & $20,-5,80,-5,40$ & $100,30,30,30,200$ & $250,250,250,250,250250,250,250,250,250$ \\
\hline L3C & $0,0,0,0,0$ & $20,-5,80,-5,40$ & $100,30,30,30,200$ & $250,250,250,250,250250,250,250,250,250$ \\
\hline
\end{tabular}

Fig. 8. The example of main(P, Q, R, S, T) parameters. Each cell has five values and represents the parameters of $\mathrm{P}, \mathrm{Q}, \mathrm{R}, \mathrm{S}, \mathrm{T}$ waves. All values are scaled to larger ones as in the professional ECG equipment.

In many cases the ECG data are not stored because there is no time to save digitalized files from examination when a patient is in a very hard condition. On the other hand, modern solutions like telemedicine system of communication between the rescue teams and diagnostic centers in hospitals allow to create databases of unique ECG signals. A well designed simulation system can create various ECG signals and can be used by specialists for data storage or the medicine students to practice knowledge about serious heart diseases. In some cases the model can be used as a compression one, because there is no satisfactory method for the ECG signal compression. Each signal may be described using only about 30 parameters to show important parts of the ECG examination.

\section{The applications for pathology detection}

There are the applications which help the cardiologists in work with the data obtained from the ECG equipment. Data stored in equipment are accessible only by dedicated software. Fortunately, software can decode signals to files with integer values of voltage. It allows to load and analyze data by exterior programs keeping regularity. For computing original signals taken from equipment, the decoding key is needed. Without the key, created by the equipment authors, data can be falsified[5]. Software which is given with equipment can not analyze the ECG signal. The ECG Holter can store data from twenty four hours and more. It is hard to analyze the data from a whole day. It consumes time and money and may cause problems with 
correct diagnosis. So the authors decided to create application to help cardiology specialists in data analysis.

The ECG Viewer is the first module of application. It allows to load data from 24h Holter with $100 \mathrm{~Hz}$ frequency. There are about 24 million values of voltage divided into three columns representing three leads of Holter ECG in every examination file. Running application can result in memory problems. That is way all data are divided into one minute segments. This solution not only simplifies memory problems while computing but gives cardiologists a full picture of heart activity at different time during the day. The application is directly destined to three leads of electrocardiographs.

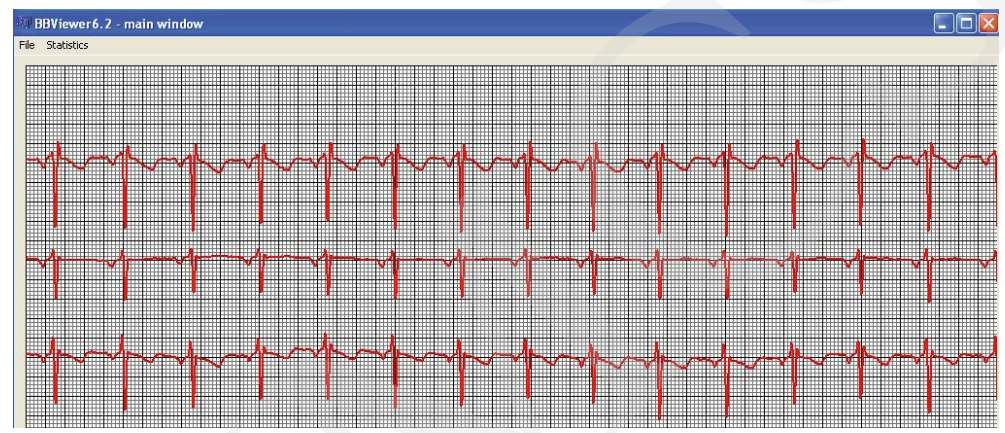

Fig. 9. Three leads of signal from the ECG Holter.

The pulse is one of the most important ECG features. It shows information describing the distance between the two successive R peaks. Every minute the average pulse is computed by the application. The differences between pulse values show bad heart activity or artefacts. This fragment of a signal can be jumped to, directly into the required part without wasting time with minute after minute view. In case of regular signals, the differences between the obtained pulse values are similar.

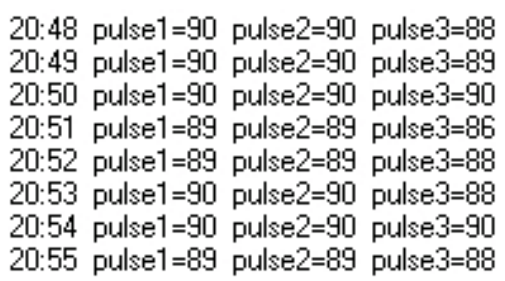

Fig. 10. Pulse results. The extract from the application contains the computed pulse, and the columns representing: time and pulse for each lead.

Unfortunately, not all signals are as regular as in Fig. 1. There are some heart pathologies which are represented by completely irregular ECG. The pulse algorithm is sometimes useless. On the other hand, in almost all cases at least one pulse result represented the values which could be classified as a good one (Fig. 3). 
$16: 47$ pulse1=68 pulse2=68 pulse $3=38$

$16: 48$ pulse $1=53$ pulse $2=9$ pulse $3=27$

$16: 49$ pulse $1=49$ pulse $2=9$ pulse $3=27$

$16: 50$ pulse $1=46$ pulse $2=9$ pulse $3=9$

$16: 51$ pulse $1=67$ pulse $2=68$ pulse $3=66$

$16: 52$ pulse1 $=53$ pulse $2=10$ pulse $3=39$

$16: 53$ pulse $1=48$ pulse $2=9$ pulse $3=27$

$16: 54$ pulse1 $=50$ pulse $2=9$ pulse $3=9$

Fig. 11. In this case only the pulse from the lead one represents correct values.

\section{The automation process}

The aim of the research is to demonstrate that every ECG signal can be described as a collection of several variables. This is a step which can be used in compression methods for better and faster data acquisition and transmission. The automation process of generating the results is complicated and hard to implement. As common statistical methods can not be used for the data comparison between the actual and simulated signals the authors want to include some limitations in the results estimation. In most cases in a ECG signal the baseline which is randomly characterized does not have an influence on patient's heart condition because only waves have. So if algorithms efficiency was tested, only waves would be analyzed. Furthermore, the process of waves detection is also important. There exist some methods for the QRS complex detection and T wave detection, but $\mathrm{P}$ wave is often not detected due to its low amplitude and short duration. That is why in some cases only manual recognition can give real chances for a wave detection.

The "So and Chan" and "Tompkins" algorithms give good chances for wave detection. The "Modified So and Chan" algorithms consist of few steps which can provide satisfactory results [1][2]. The digital band-pass filter reduces noise. If the low-pass filter is described by:

$$
H(z)=\frac{\left(1-z^{-6}\right)^{2}}{\left(1-z^{-1}\right)^{2}}
$$

and difference equation is:

$$
y(n T)=2 y(n T-T)-y(n T-2 T)+x(n T)-2 x(n T-6)+x(n T-12 T)
$$

and high-pass filter:

$$
H(z)=\frac{-1+32 z^{-16}+z^{-32}}{\left(1-z^{-1}\right)}
$$

and its difference equation is:

$$
y(n T)=32 x(n T-16 T)-[y(n T-T)+x(n T)-x(n T-32 T)] .
$$

Then the signal slope calculation is used: 
Pobrane z czasopisma Annales AI- Informatica http://ai.annales.umcs.pl

Data: 26/04/2023 16:44:03

$$
\text { Signal_slope }(n)=-2 X(n-2)-X(n-1)+X(n+1)+2 X(n+2) \text {. }
$$

46 sets of ECG signals taken from the MIT-BIH database give the average $95 \%$ so these algorithms can be used for detection of different types of QRS complexes.

\section{The results}

Currently the best way for estimation of the results is the scanned comparison of the signals from the original examinations with the simulated ones.
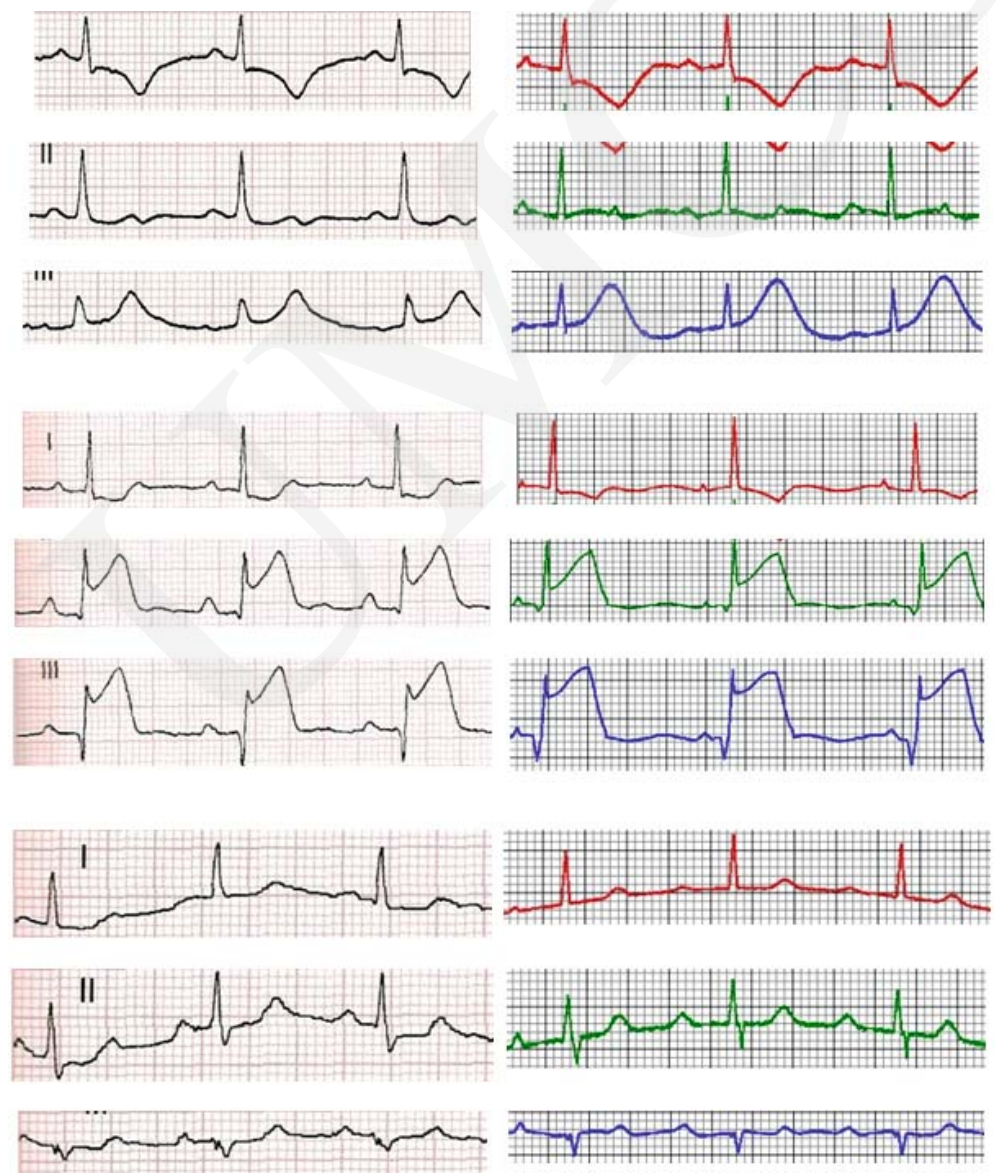

Fig. 4. On the left, actual ECG's are given, on the right those simulated by the authors.

As it can be observed, in many cases the simulated signal is very similar to the original one. Some of them have small shape changes but all clinical information is fulfilled. STEMI modifications, waves' amplitudes and duration time, distances between the successive peaks 
and the baseline characters indicates that the algorithms can be applied for fast ECG signal description.

\section{References}

[1] Chuang-Chien Chiu, Tong-Hong Lin, Ben-Yi Liau, Using correlation coefficient in ECG waveform for arrhythmia detection, Center for Biomedical Engineering 17(3) (2005): $147-152$.

[2] Ren-Guey Lee, I-Chi Chou, Chien-Chin Lai, Ming-Hsiu Liu, Ming-Jang Chiu, A novel QRS detection algorithm applied to the analysis for heart rate variability of patient with sleep apnea. Institute of Computer and Communication Engineering, National Taipei University of Technology 17(5) (2005).

[3] Hualao Ling, Qiu-Hua Lin and J.D.X.Chen, Application of the Empirical Mode decomposition to the analysis of Esophageal Reflux Disease, IEEE transactions on Biomedical Engineering 52(10) (2005).

[4] Macfarlane P. W., Age, sex, and the ST amplitude in health and disease, J. Electrocardiology 34(supplement) (2001): 235-410.

[5] Nugent C. D, Webb J. A. C, Black N. D. et al., An intelligent framework for the classification of the 12-lead ECG, Artificial Intelligence in Medicine 16 (1999): 205-222. 